\title{
Effect of masseter muscle activity during wakefulness and sleep on tooth wear
}

\author{
Keisuke Kitagawa, Naoki Kodama *, Yousuke Manda, Keitaro Mori, Hiroshi Furutera, \\ Shogo Minagi
}

Department of Occlusal and Oral Functional Rehabilitation, Graduate School of Medicine, Dentistry and Pharmaceutical Sciences, Okayama University, Japan

\begin{abstract}
Purpose: To investigate the effect of masseter muscle activity during wakefulness and sleep on tooth wear.

Methods: Sixteen participants with no or mild tooth wear (NMTW group) and sixteen participants with moderate-tosevere tooth wear (MSTW group) were enrolled. The severity of tooth wear was evaluated using the occlusal and incisal indices of the tooth wear index. Surface electromyography was performed to record the electrical activity of the left masseter muscle during wakefulness and sleep. Electromyographic activity was detected using an electromyographic threshold of $5 \%$ and $20 \%$ of maximal voluntary clenching (MVC). The total duration of electromyographic activity and bruxism episodes were calculated.

Results: The mean ages of the NMTW and MSTW groups were $71.75 \pm 7.61$ years and $71.69 \pm 7.49$ years, respectively. The mean cumulative duration of electromyographic activity during wakefulness using a threshold of $>5 \%$ MVC was $6.44 \pm$ $4.52 \mathrm{~min} / \mathrm{h}$ and $13.62 \pm 10.08 \mathrm{~min} / \mathrm{h}$ for the NMTW and MSTW groups, respectively $(p=0.048)$. The mean total durations of electromyographic activity during wakefulness and sleep using a threshold of $>20 \%$ MVC were $1.08 \pm 1.70 \mathrm{~min} / \mathrm{h}$ and $1.05 \pm 3.02 \mathrm{~min} / \mathrm{h}$, respectively, in the NMTW group and $4.78 \pm 6.37 \mathrm{~min} / \mathrm{h}$ and $1.61 \pm 1.79 \mathrm{~min} / \mathrm{h}$, respectively, in the MSTW group ( $p=0.048$ and $p=0.003$, respectively).

Conclusion: These results suggest that masseter electromyographic activity during wakefulness and sleep may be related to the severity of tooth wear.
\end{abstract}

Keywords: Tooth wear, Awake bruxism, Sleep bruxism, Masseter muscle, Surface electromyogram

Received 8 July 2021, Accepted 18 October 2021, Available online 24 December 2021

\section{Introduction}

Tooth wear, caused by attrition, erosion, and abrasion, is regarded as the surface loss of dental hard tissues[1]. Although tooth wear might result from any one of these processes, it is generally considered a multifactorial process[2]. Previous studies have reported that tooth wear affects not only the oral cavity, such as a decrease in masticatory function[3] and oral health-related quality of life[4], but also affects the whole body, such as a decrease in bone density[5] and worsening of diabetes[6]. Additionally, tooth wear is associated with sleep disorders, orofacial pain, oral dryness, gastroesophageal reflux disease, and sleep bruxism[7].

Among the different types of tooth wear, the attrition type of tooth wear is featured by matching wear facets between opposing teeth and is reported to be associated with sleep bruxism[8]. Since sleep and awake bruxism are independent events that occur during sleep and wakefulness, respectively[9], they should be distinguished

DOI: https://doi.org/10.2186/jpr.JPR_D_21_00171

*Corresponding author: Naoki Kodama, Department of Occlusal and Oral Functional Rehabilitation, Okayama University Graduate School of Medicine, Dentistry and Pharmaceutical Sciences, 2-5-1 Shikata-cho, Kita-ku, Okayama 700-8525, Japan.

E-mail address: naoki-k@md.okayama-u.ac.jp

Copyright: @ 2021 Japan Prosthodontic Society. All rights reserved. accordingly. Sleep bruxism is characterized by rhythmic and nonrhythmic masticatory muscle activity during sleep, whereas awake bruxism is a masticatory muscle activity characterized by repetitive or sustained tooth contact and/or bracing or thrusting of the mandible during wakefulness[10]. The current gold standard method for the diagnosis of sleep bruxism is polysomnography with audiovisual recording[11]. However, polysomnography has some disadvantages in terms of cost, analysis time, laboratory determination, and skillful analysis[12,13].

Recent advances in wearable devices have enabled the evaluation of awake bruxism[14-16]; electromyographic recordings can be used to diagnose awake bruxism during wakefulness[10]. Although previous studies have evaluated the association between sleep bruxism and tooth wear using a portable electromyographic measurement system, the relationship between them remains unclear[8]. Additionally, no study has been conducted on the association between awake bruxism and tooth wear. This study aimed to assess masseter electromyographic activity during wakefulness and sleep in individuals with moderate or severe tooth wear compared to an age- and sex-matched control group with no or mild tooth wear. We hypothesized that there was no significant difference in masseter electromyographic activity during wakefulness and sleep between the two groups. 
(a)

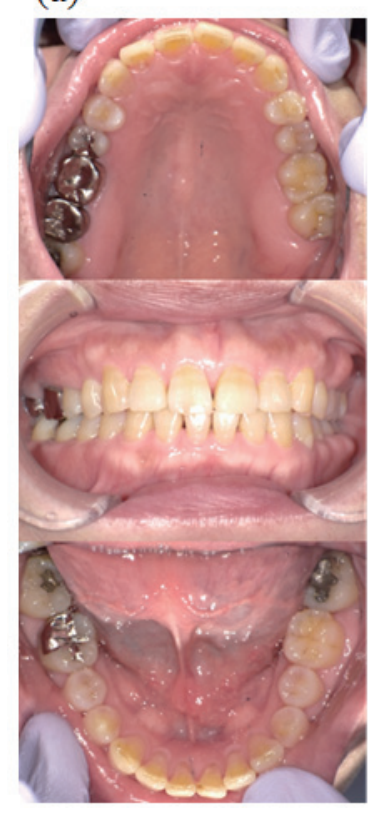

(b)

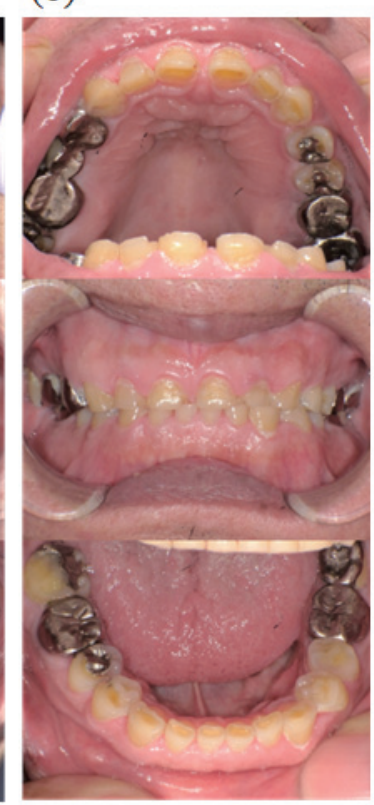

Fig. 1. Intraoral findings of the participants. (a) An example of oral photographs in the no or mild tooth wear (NMTW) group. (b) An example of oral photographs in the moderate-to-severe tooth wear (MSTW) group.

\section{Material and Methods}

\subsection{Participants}

This study was conducted between December 2018 and December 2020. Participants were recruited from among the outpatients of the Clinical Division of Occlusion and Removable Prosthodontics at the Okayama University Hospital. All participants signed an informed consent document prior to the experiment. The study protocol was approved by the Ethics Committee of the Okayama University Hospital (No. KEN1810-001). Two groups were recruited: those with moderate-to-severe tooth wear (MSTW group) and those with no or mild tooth wear (NMTW group) (Fig. 1).

The severity of tooth wear was assessed using the occlusal and incisal indices of the tooth wear index (TWI)[17]. The evaluation method was as follows: 0 , no loss of enamel; 1, loss of enamel; 2 , exposure of dentin less than one-third of the occlusal surface or exposed dentin at the incisal edge; 3 , exposure of dentin more than one-third of the occlusal surface or severely exposed dentin without exposure of the dental pulp or secondary dentin; and 4, complete loss of occlusal enamel, exposed dental pulp or secondary dentin at the occlusal/incisal area. The TWI consists of five levels from 0-4, and the higher the number of the TWI, the greater the severity of tooth wear[18]. One examiner (K.K.) recorded the TWI scores on the occlusal or incisal surfaces of all teeth, except for those undergoing restoration or prosthetic treatment.

The MSTW group consisted of 16 participants. The inclusion criteria for the MSTW group were: i) an average TWI score $\geq 2$ and ii) one or more occlusal supporting areas on the molars by natural teeth or fixed prosthesis (Eichner Index A1-A3 or B1-B3)[19]. Similar to the MSTW group, the NMTW group consisted of 16 participants. The inclusion criteria for the NMTW group were: i) an average TWI score < 2 ; ii) one or more occlusal supporting areas on the molars by natural teeth or fixed prosthesis (Eichner Index A1-A3 or B1-B3); and iii) sexmatched to the MSTW group and up to one year of age difference with each participant in the MSTW group. Removable denture wearers were included in both groups if the inclusion criteria (number ii) were satisfied.

The exclusion criteria for the MSTW and NMTW groups were as follows: i) a history of orthodontic treatment, ii) a history of reflux esophagitis, iii) frequent consumption of acidic foods, iv) systemic diseases related to tooth wear, or v) temporomandibular disorders.

The sample size of 15 participants in each group was determined from the results of a previous study[20]. The calculation was performed using $G^{*}$ Power version 3.1.7 $[a=0.05,(1-\beta)=0.8$, effect size $=0.8]$.

\subsection{Electromyographic Data recording}

Interviews with the participants confirmed that they met all the inclusion criteria and none of the exclusion criteria. Prior to electrode attachment, the skin was wiped with $70 \%$ ethyl alcohol. The electrode was attached to the left masseter muscle as described in previous studies[15]. Electromyographic recordings were performed using differential surface electrodes with three disposable $\mathrm{Ag} / \mathrm{AgCl}$ surface electrodes (SMP-300, Mets) with center-to-center distances of $8 \mathrm{~mm}$. The electrodes were secured to the buccal skin using a thin biocompatible adhesive tape (Cathereep FS 1010; Nichiban Co. Ltd., Tokyo, Japan). Electromyographic recordings were performed throughout the day and night (from one morning to the next) until the participants woke up. The hardware used for the electromyographic recording consisted of an analog signal processing and differential amplification integrated hybrid circuit (NB-6201HS; Nabtesco Co., Kobe, Japan), which included $10 \mathrm{~Hz}$ high-pass and $1000 \mathrm{~Hz}$ low-pass filters, and a two-channel digital recorder (ICR-PS004 M; Sanyo Electric Co., Ltd., Osaka, Japan). A voice-operated trigger switch was used with a condenser microphone attached to the skin on the neck adjacent to the larynx to distinguish surface electromyographic activity during speech and other electromyographic activity, and the signal was recorded on the second channel of a two-channel digital recorder. After completing the ambulatory surface electromyographic recording hardware, participants were instructed to perform maximal voluntary clenching (MVC) three times for $2 \mathrm{~s}$ at intervals of $2 \mathrm{~s}$. Of the three MVC actions, the highest signal was regarded as $100 \%$ MVC. Participants were instructed to fill in their mealtime, bedtime, and wake-up time on the dedicated record table, which was mailed to our department the next day. After the explanation of the electromyographic recording, participants left the hospital and resumed their daily lives. Participants wearing removable partial dentures were instructed to remove them during sleep.

\subsection{Data analysis}

Data processing and analysis were performed in the same manner as previously described[15]. The recorded electromyographic data in the MP3 format were processed offline, converted into a resource interchange file format of waveform audio (WAV), filtered using a notch filter $(60 \mathrm{~Hz})$, and then down sampled to $100 \mathrm{~Hz}$. The masseter electromyographic activity accompanied by positive voice-operated trigger signals was regarded as that during speech. The total duration of masticatory electromyographic bursts using 
Table 1. Parameter tables for mean age, number of remaining teeth, mean value of tooth wear index (TWI), number of participants, and Eichner's classification

\begin{tabular}{lccc}
\hline & NMTW group & MSTW group & $p$ \\
\hline Mean Age & $71.75 \pm 7.61$ & $71.69 \pm 7.49$ & 0.98 \\
Number of Remaining Teeth & $23.94 \pm 3.73$ & $22.44 \pm 3.14$ & 0.23 \\
Mean of TWI Score & $1.44 \pm 0.24$ & $2.83 \pm 0.54$ & $<0.001$ \\
Number of Participants & 16 (M:11 W:5) & 16 (M:11 W:5) & \\
Number of Eichner's classification A1 & 4 & 1 & \\
Number of Eichner's classification A2 & 2 & 2 & \\
Number of Eichner's classification A3 & 0 & 3 & \\
Number of Eichner's classification B1 & 4 & 2 & \\
Number of Eichner's classification B2 & 5 & 3 & \\
Number of Eichner's classification B3 & 1 & 5 & \\
\hline
\end{tabular}

M=Man, W=Woman
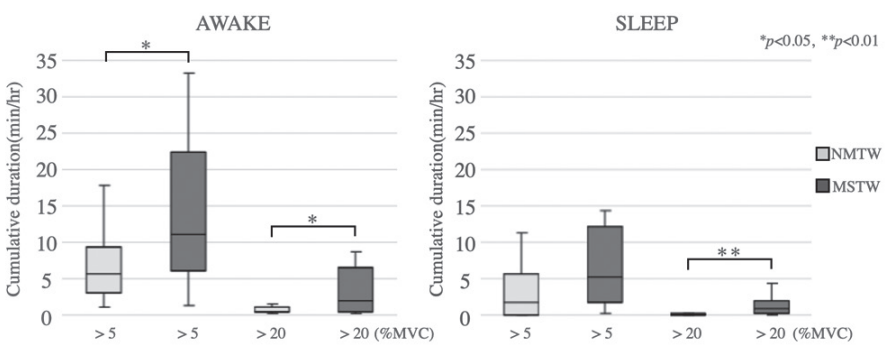

Fig. 2. Duration of masseter muscle activity per hour. The mean cumulative duration of masseter muscle activity during wakefulness with intensities of $>5 \%$ and $>20 \%$ maximal voluntary clenching (MVC) for the MSTW group is significantly longer than that of the NMTW group. In contrast, the mean cumulative duration of masseter muscle activity during sleep with intensities of not $>5 \%$ but $>20 \%$ MVC for the MSTW group is significantly longer than that of the NMTW group.
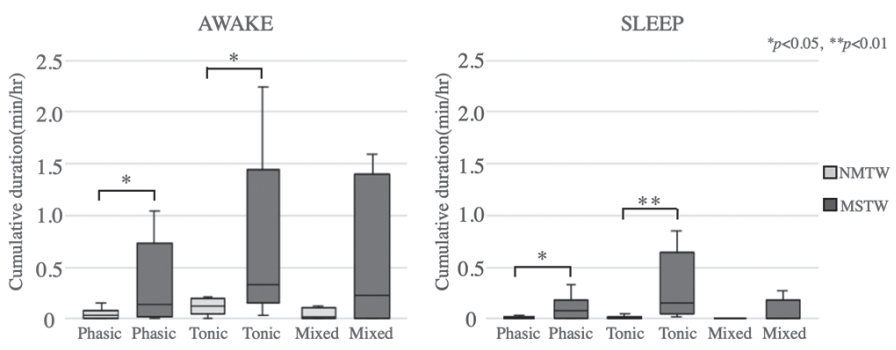

Fig. 3. Duration of phasic, tonic, and mixed episodes per hour (threshold: $>20 \%$ MVC). Phasic and tonic episodes are significantly longer in the MSTW group than in the NMTW group during wakefulness as well as sleep.

thresholds of $5 \%$ and $20 \%$ MVC was calculated. Phasic episodes, tonic episodes, and mixed episodes were analyzed using a threshold of $20 \%$ MVC according to a previous study[21]. Each episode was calculated separately and divided into wakefulness and sleep. All electromyographic data were analyzed in a blinded condition so that the TWI score of each participant was undisclosed.

\subsection{Statistical analysis}

The Shapiro-Wilk test was used to test the normality of the data. Descriptive statistics are represented as the mean \pm standard devia- tion for continuous variables and frequency. Welch's t-test was used to compare the variables with a normal distribution. The Mann-Whitney $U$ test was used to compare continuous variables with a nonnormal distribution. The level of significance was set at $5 \%(p<0.05)$ in this study. All data analysis were performed using a statistical software package (BellCurve for Excel, Social Survey Research Information Co., Ltd, Tokyo, Japan).

\section{Results}

The participant characteristics are presented in Table 1. The mean ages of the NMTW and MSTW groups were $71.75 \pm 7.61$ years and $71.69 \pm 7.49$ years, respectively $(p=0.98)$. The mean TWI score of the MSTW group $(2.83 \pm 0.54)$ was significantly higher than that of the NMTW group $(1.44 \pm 0.24)(p<0.001)$. The mean number of remaining teeth was $23.94 \pm 3.73$ in the NMTW group and $22.44 \pm 3.14$ in the MSTW group $(p=0.23)$. The total duration of the masticatory electromyographic activity, except chewing and speech, was analyzed separately for wakefulness and sleep (Fig. 2). The total durations of masticatory electromyographic activity during wakefulness using a threshold of $5 \%$ MVC were $6.44 \pm 4.52 \mathrm{~min} / \mathrm{h}$ and $13.62 \pm 10.08 \mathrm{~min} / \mathrm{h}$ for the NMTW and MSTW groups, respectively ( $p=0.048$ ). The mean total durations of masticatory electromyographic activity during sleep using a threshold of 5\% MVC were $8.08 \pm 15.68 \mathrm{~min} / \mathrm{h}$ and $9.56 \pm$ $12.58 \mathrm{~min} / \mathrm{h}$ for the NMTW and MSTW groups, respectively $(p=0.109)$. The mean total durations of masticatory electromyographic activity during wakefulness and sleep using a threshold of 20\% MVC were $1.08 \pm 1.70 \mathrm{~min} / \mathrm{h}$ and $1.05 \pm 3.02 \mathrm{~min} / \mathrm{h}$, respectively, in the NMTW group, and $4.78 \pm 6.37 \mathrm{~min} / \mathrm{h}$ and $1.61 \pm 1.79 \mathrm{~min} / \mathrm{h}$, respectively, in the MSTW group ( $p=0.048$ and $p=0.003$, respectively).

Figure 3 shows the total duration of phasic, tonic, and mixed electromyographic activity per hour using a threshold $>20 \%$ MVC. The MSTW group had significantly longer phasic and tonic episodes than the NMTW group ( $p=0.042$ and $p=0.028$, respectively). Phasic and tonic episodes were significantly longer in the MSTW group than in the NMTW group during sleep $(p=0.027$ and $p=0.001$, respectively); however, there was no significant difference between the two groups during mixed episodes of wakefulness and sleep ( $p=0.110$ and $p=0.104$, respectively). The receiver operating characteristic (ROC) curve was used to estimate the cut-off value needed to diagnose severe tooth wear. Figure 4 shows the ROC curve for distinguishing participants in the MSTW group from the NMTW group based on the total duration of masticatory electromyographic activity per hour using thresholds of 5\% MVC and > 20\% MVC. According to the ROC curve analysis, the optimal cutoff point at a threshold of $20 \%$ MVC for awake bruxism was $1.115 \mathrm{~min} / \mathrm{h}$ (sensitivity=0.688, specificity $=0.750$ ) and that for sleep bruxism was $0.196 \mathrm{~min} / \mathrm{h}$ (sensitivity $=0.875$, specificity $=0.688$ ).

\section{Discussion}

This study examined the association between masseter electromyographic activity during wakefulness and sleep in individuals with MSTW compared with an age- and sex-matched control group with NMTW. Our results suggested that masseter electromyographic activity increased during wakefulness and sleep as the severity of tooth wear was progressed. Therefore, the null hypothesis was rejected. To the best of our knowledge, this is the first case-control study to demonstrate an association between higher masseter electromyographic activity during wakefulness and sleep and increased severity of tooth wear. In particular, no study has scientifically assessed 

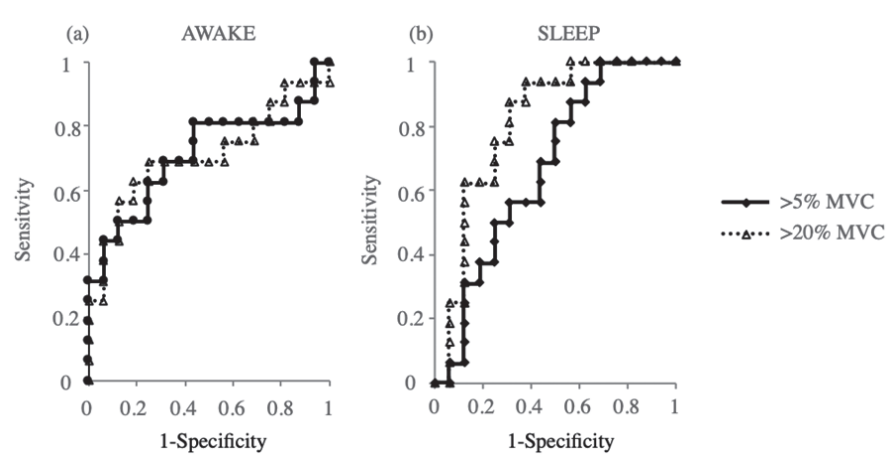

Fig. 4. Receiver Operating Characteristic (ROC) curve. (a) ROC curve during wakefulness. (b) ROC curve during sleep.

the relationship between awake bruxism and tooth wear due to the restricted environment of polysomnography, which is the gold standard method for the diagnosis of bruxism. However, our portable device enabled the measurement of masseter electromyographic activity during wakefulness and sleep with high accuracy.

The mean duration of masticatory electromyographic activity per hour using a threshold of $20 \%$ and $5 \%$ MVC during wakefulness was significantly longer in the MSTW group than in the NMTW group ( $p=0.048, p=0.048$ respectively). This indicated a significant association between awake bruxism and the severity of tooth wear, regardless of the intensity of masseter electromyographic activity. Interestingly, as shown in Figure 2, masseter electromyographic activity during wakefulness with intensities of $>5 \%$ MVC was significantly different between the two groups $(p=0.048)$. The masseter electromyographic activity during wakefulness was statistically significant, although there was no significant difference in masseter electromyographic activity during sleep. Given these results, awake bruxism, especially with a low intensity of masseter electromyographic activity during wakefulness, cannot be underestimated.

Despite the intensities of electromyographic activity, awake bruxism has also gained attention in recent years. Our electromyographic recording system is characterized by an electrode attachment only to the left masseter muscle so as not to interfere with daily activities and a voice-operated exchange trigger to eliminate conversation instead of an electrode attached to the right masseter muscle. In addition, this recording system had a high $\mathrm{S} / \mathrm{N}$ ratio, which could be useful for electromyography, especially for the evaluation of long-sustained low-level electromyographic activity, as stated in our previous study[21]. Therefore, our electromyographic recording system enabled the evaluation of the relationship between masseter electromyographic activity during wakefulness, sleep, and pathological conditions, such as periodontal disease[15] and occlusal bite collapse[16].

Many dentists have long believed that matching the wear facets between opposing teeth is an important factor for the clinical diagnosis of sleep bruxism. As tooth wear is a cumulative record of both functional and parafunctional activities, its evaluation to establish a diagnosis between current sleep bruxism and the severity of tooth wear remains controversial[22]. Some studies have reported a positive relationship between tooth wear and self-reported bruxism[23-25]. Previous studies using polysomnography showed that tooth wear was associated with a higher frequency of rhythmic mas- ticatory muscle activity; however, it was not associated with awake bruxism[26,27]. The relationship between sleep bruxism and tooth wear in this study was similar to that reported in previous studies. Previous studies reported no significant correlation between the severity of tooth wear and sleep bruxism[8,28].

Our study mainly included older individuals. The mean number of remaining teeth was more than 22 in the NMTW and MSTW group individuals. One of the inclusion criteria for both groups in this study was one or more occlusal supporting areas on the molars (Eichner Index A1-A3 or B1-B3), which indicated that the number of remaining teeth was valid considering the occlusal support. In a previous observational study targeting healthy older Japanese adults, the mean number of remaining teeth was $21.1 \pm 0.6[29]$. Furthermore, another study showed that the number of functional teeth in the decreased posterior occlusal area for older Japanese individuals was $22.2 \pm$ 3.5[30]. Our results are consistent with these findings, although the number of remaining teeth may be unusual for their age.

In general, sleep bruxism reduces with age[7,31-33], although our results were inconsistent with this statement. This could be because the results may depend on the differences in the age group and the severity of tooth wear. Our study suggests that patients with moderate-to-severe tooth wear may be less susceptible to age-related reductions in sleep bruxism. Therefore, such patients may show higher sleep bruxism events even when they are older than those with no or mild tooth wear.

In this study, we used the TWI to evaluate the severity of tooth wear. As TWI is a simple evaluation method, it is often used in many studies. Some studies reported appointing a single evaluator for assigning the TWI score[6,34], whereas others did not report the number of evaluators used[4,35]. The evaluator of the TWI score in this study was fully trained in advance, but there might have been differences in the TWI score if there were multiple evaluators. The TWI scores in this study were used to classify the participants into the two study groups. As shown in Table 1, there was a significant difference in the TWI scores between the two groups. Thus, there was no possibility that the group to which participants belonged would have changed regardless of the number of evaluators, although there might have been slight differences in the TWI scores assigned. Therefore, having only one evaluator to assign the TWI score was considered appropriate for this study.

Differences in the indices used to assess tooth wear might affect the results of a study. In a previous study using electromyography and accelerometry to identify patterns of masseter muscle activity during sleep, masseter muscle electromyographic activity was classified into three patterns: tapping, clenching, and grinding; the mean values of muscle activity were $2.6 \%, 19.7 \%$, and $32.8 \%$ MVC, respectively[36]. This classification suggested that in the MSTW group, weak muscle activity during wakefulness, equivalent to tapping, was observed more commonly than in the NMTW group. However, many participants were older, and this result may not apply to younger individuals.

In this study, the intensities of $>5 \%$ and $>20 \%$ MVC were used as thresholds for the evaluation of masseter electromyographic activity; however, the maximum occlusal force was not considered. The occlusal force has been reported to increase with age; it remains nearly constant between the ages of 20 and 40 or 50 years and then decreases[37]. Patients with severe tooth wear are reported to have 
a lower electromyographic activity of the mandibular elevation muscle during maximal voluntary clenching than healthy patients[35]. Most of the participants in this study were older, indicating the possibility of a reduction in occlusal force and the rate of tooth wear.

In this study, electromyographic recordings were performed for only one day, indicating that the first night effect could not be eliminated. However, a study comparing rhythmic masticatory muscle activity (RMMA) during the first night with that of the second night using polysomnography showed no first-night effect on the severity of RMMA frequency[38]. Additionally, the first night effect was not observed in a study that evaluated the masseter electromyographic activity over 4 consecutive nights using a portable device[39]. Furthermore, Saito et al. investigated the minimum measurement time of masseter electromyographic activity needed to assess awake bruxism during wakefulness and concluded that more than to 2.53.0 $\mathrm{h}$ were necessary for accurately measuring muscle activity[14]. Based on these results, a measurement time of approximately $1 \mathrm{~d}$ was considered adequate.

This study has some limitations. First, the number of participants in this study was small. Although the number of participants was set to be larger than the sample size in consideration of dropout in most clinical trials, all participants agreed to participate in this study. Additionally, as this was an observational study rather than an interventional study, it was possible to recruit participants as needed. Considering this fact, the number of participants in this study was adequate, although the sample size was small. Second, a history of reflux esophagitis and frequent consumption of acidic foods was confirmed only by inquiry. According to an in vitro study using extracted teeth, an increase in dental attrition affecting the enamel was observed at a $\mathrm{pH}$ of 4.8, but not at $\mathrm{pH} 5.5[40]$. Tooth wear of the enamel may be greatly influenced by bruxism under low $\mathrm{pH}$ conditions, and further investigation is needed. Third, the criteria used for the evaluation of tooth wear were subjective and not objective. TWI is widely used as a method for evaluating tooth wear, although it cannot be quantified. Therefore, in recent years, intraoral scanners have been used to evaluate the degree of tooth wear[41-43]. The use of intraoral scanners and other methods to quantitatively assess the degree of occlusal wear may enable future longitudinal studies. Finally, we could not evaluate tooth hardness. In addition, the participants had several prostheses. Tooth wear is related to the hardness of the tooth and the type of prosthesis used[44]. Taking into consideration the effect of prostheses on tooth wear, the TWI score was evaluated except for the teeth with prostheses. However, it is of great significance that the difference in TWI scores affects masseter electromyographic activity during wakefulness and sleep, especially during wakefulness, regardless of the intensity. Since it is difficult to evaluate the hardness of teeth, further studies targeting participants with natural dentition without any prosthesis are needed to evaluate the effectiveness of tooth wear on awake bruxism and sleep bruxism.

\section{Conclusion}

Within the limitations of this study, our results suggest that masseter muscle activity during wakefulness and sleep may be related to the severity of tooth wear. Thus, the assessment of masticatory electromyographic activity during wakefulness and sleep could be an important predictor of the progression of tooth wear.

\section{Conflicts of interest}

The authors declare no conflicts of interest.

\section{References}

[1] Mehta SB, Banerji S, Millar BJ, Suarez-Feito JM. Current concepts on the management of tooth wear: part 1. Assessment, treatment planning and strategies for the prevention and the passive management of tooth wear. Br Dent J. 2012;212:17-27. https://doi.org/10.1038/sj.bdj.2011.1099, PMID:22240686

[2] Bartlett D, Dugmore C. Pathological or physiological erosion-is there a relationship to age? Clin Oral Investig. 2008;12(suppl 1):27-31. https://doi. org/10.1007/s00784-007-0177-1, PMID:18228061

[3] Muts EJ, van Pelt H, Edelhoff D, Krejci I, Cune M. Tooth wear: A systematic review of treatment options. J Prosthet Dent. 2014;112:752-9. https://doi. org/10.1016/j.prosdent.2014.01.018, PMID:24721500

[4] Sterenborg BAMM, Bronkhorst EM, Wetselaar P, Lobbezoo F, Loomans BAC, Huysmans MCDNJM. The influence of management of tooth wear on oral health-related quality of life. Clin Oral Investig. 2018;22:2567-73. https://doi. org/10.1007/s00784-018-2355-8, PMID:29397468

[5] Sierpinska T, Konstantynowicz J, Orywal K, Golebiewska M, Szmitkowski M. Copper deficit as a potential pathogenic factor of reduced bone mineral density and severe tooth wear. Osteoporos Int. 2014;25:447-54. https://doi. org/10.1007/s00198-013-2410-x, PMID:23797848

[6] Srisilapanan $P$, Jindarat $M$, Roseman J. The prevalence and severity of tooth wear in Type 2 diabetic patients. Int J Dent. 2018;2018:1-5. https://doi. org/10.1155/2018/3608158, PMID:30651731

[7] Wetselaar P, Manfredini D, Ahlberg J, Johansson A, Aarab G, Papagianni $\mathrm{CE}$, et al. Associations between tooth wear and dental sleep disorders: $\mathrm{A}$ narrative overview. J Oral Rehabil. 2019;46:765-75. https://doi.org/10.1111/ joor.12807, PMID:31038764

[8] Jonsgar C, Hordvik PA, Berge ME, Johansson AK, Svensson P, Johansson A. Sleep bruxism in individuals with and without attrition-type tooth wear: an exploratory matched case-control electromyographic study. J Dent. 2015;43:1504-10. https://doi.org/10.1016/j.jdent.2015.10.002, PMID:26455540

[9] Lavigne GJ, Khoury S, Abe S, Yamaguchi T, Raphael K. Bruxism physiology and pathology: an overview for clinicians. J Oral Rehabil. 2008;35:476-94. https://doi.org/10.1111/j.1365-2842.2008.01881.x, PMID:18557915

[10] Lobbezoo F, Ahlberg J, Raphael KG, Wetselaar P, Glaros AG, Kato T, et al. International consensus on the assessment of bruxism: report of a work in progress. J Oral Rehabil. 2018;45:837-44. https://doi.org/10.1111/joor.12663, PMID:29926505

[11] Lobbezoo F, Ahlberg J, Glaros AG, Kato T, Koyano K, Lavigne GJ, et al. Bruxism defined and graded: an international consensus. J Oral Rehabil. 2013;40:2-4. https://doi.org/10.1111/joor.12011, PMID:23121262

[12] Farella M, Palla S, Gallo LM. Time-frequency analysis of rhythmic masticatory muscle activity. Muscle Nerve. 2009;39:828-36. https://doi.org/10.1002/ mus.21262, PMID:19306326

[13] Gallo LM, Lavigne G, Rompré P, Palla S. Reliability of scoring EMG orofacial events: polysomnography compared with ambulatory recordings. J Sleep Res. 1997;6:259-63. https://doi.org/10.1111/j.1365-2869.1997.00259.x, PMID:9493526

[14] Saito T, Yamaguchi T, Mikami S, Saito M, Nakajima T, Yachida W, et al. Minimum measurement time of masseteric electromyogram required for assessment of awake bruxism during the daytime. Cranio. 2019;19:1-8. https://doi.org/10.1080/08869634.2019.1704982, PMID:31856680

[15] Kato S, Ekuni D, Kawakami S, Mude AH, Morita M, Minagi S. Relationship between severity of periodontitis and masseter muscle activity during waking and sleeping hours. Arch Oral Biol. 2018;90:13-8. https://doi.org/10.1016/j. archoralbio.2018.02.021, PMID:29524787

[16] Kawakami S, Kumazaki Y, Manda Y, Oki K, Minagi S. Specific diurnal EMG activity pattern observed in occlusal collapse patients: relationship between diurnal bruxism and tooth loss progression. PLoS One. 2014;9:e101882. https://doi.org/10.1371/journal.pone.0101882, PMID:25010348

[17] Smith BG, Knight JK. An index for measuring the wear of teeth. Br Dent J. 1984;156:435-8. https://doi.org/10.1038/sj.bdj.4805394, PMID:6590081

[18] Wetselaar P, Faris A, Lobbezoo F. A plea for the development of an universally accepted modular tooth wear evaluation system. BMC Oral Health. 2016;16:115. https://doi.org/10.1186/s12903-016-0309-6, PMID:27809895 
[19] Eichner K. [Renewed examination of the group classification of partially edentulous arches by Eichner and application advices for studies on morbidity statistics]. Stomatol DDR. 1990;40:321-5. PMID:2270610

[20] Cohen J. (1988). Statistical Power Analysis for the Behavioral Science (2nd ed.). Lawrence Erlbaum Associates Publishers.

[21] Kumazaki Y, Naito M, Kawakami S, Hirata A, Oki K, Minagi S. Development of a speech-discriminating electromyogram system for routine ambulatory recordings for the low-level masseter muscle activity. J Oral Rehabil. 2014;41:266-74. https://doi.org/10.1111/joor.12138, PMID:24612227

[22] Velly-Miguel AM, Montplaisir J, Rompre PH, Lund JP, Lavigne GJ. Bruxism and other orofacial movements during sleep. J Craniomandib Disord Facial Oral Pain. 1992;6:71-81.

[23] Yoshida Y, Suganuma T, Takaba M, Ono Y, Abe Y, Yoshizawa S, et al. Association between patterns of jaw motor activity during sleep and clinical signs and symptoms of sleep bruxism. J Sleep Res. 2017;26:415-21. https://doi. org/10.1111/jsr.12481, PMID:27996170

[24] Carlsson GE, Johansson A, Lundqvist S. Occlusal wear: A follow-up study of 18 subjects with extensively worn dentitions. Acta Odontol Scand. 1985;43:83-90. https://doi.org/10.3109/00016358509046491, PMID:3863449

[25] Ekfeldt A, Hugoson A, Bergendal T, Helkimo M. An individual tooth wear index and an analysis of factors correlated to incisal and occlusal wear in an adult Swedish population. Acta Odontol Scand. 1990;48:343-9. https://doi. org/10.3109/00016359009033627, PMID:2251924

[26] Abe S, Yamaguchi T, Rompré PH, De Grandmont P, Chen YJ, Lavigne GJ. Tooth wear in young subjects: a discriminator between sleep bruxers and controls? Int J Prosthodont. 2009;22:342-50. PMID:19639069

[27] Yoshizawa S, Suganuma T, Takaba M, Ono Y, Sakai T, Yoshizawa A, et al. Phasic jaw motor episodes in healthy subjects with or without clinical signs and symptoms of sleep bruxism: a pilot study. Sleep Breath. 2014;18:187-93. https://doi.org/10.1007/s11325-013-0868-6, PMID:23775827

[28] Manfredini D, Lombardo L, Visentin A, Arreghini A, Siciliani G. Correlation between sleep-time masseter muscle activity and tooth wear: an electromyographic study. J Oral Facial Pain Headache. 2019;33:199-204. https:// doi.org/10.11607/ofph.2081, PMID:31013339

[29] Hasegawa Y, Sakuramoto A, Sugita H, Hasegawa K, Horii N, Sawada T, et al. Relationship between oral environment and frailty among older adults dwelling in a rural Japanese community: a cross-sectional observational study. BMC Oral Health. 2019;19:23. https://doi.org/10.1186/s12903-0190714-8, PMID:30669995

[30] Fushida S, Kosaka T, Kida M, Kokubo Y, Watanabe M, Higashiyama A, et al. Decrease in posterior occlusal support area can accelerate tooth loss: the Suita study. J Prosthodont Res. 2021;65:321-6. https://doi.org/10.2186/jpr. JPR_D_20_00005, PMID:33177306

[31] Maluly M, Andersen ML, Dal-Fabbro C, Garbuio S, Bittencourt L, de Siqueira JTT, et al. Polysomnographic study of the prevalence of sleep bruxism in a population sample. J Dent Res. 2013;92(suppl):S97-103. https://doi. org/10.1177/0022034513484328, PMID:23690359
[32] Kato T, Velly AM, Nakane T, Masuda $Y$, Maki S. Age is associated with self-reported sleep bruxism, independently of tooth loss. Sleep Breath. 2012;16:1159-65. https://doi.org/10.1007/s11325-011-0625-7, PMID:22146891

[33] Ohayon MM, Li KK, Guilleminault C. Risk factors for sleep bruxism in the general population. Chest. 2001;119:53-61. https://doi.org/10.1378/ chest.119.1.53, PMID:11157584

[34] Oginni AO, Agbakwuru EA, Ndububa DA. The prevalence of dental erosion in Nigerian patients with gastro-oesophageal reflux disease. BMC Oral Health. 2005;5:1. https://doi.org/10.1186/1472-6831-5-1, PMID:15740613

[35] Sierpinska T, Kuc J, Golebiewska M. Assessment of masticatory muscle activity and occlusion time in patients with advanced tooth wear. Arch Oral Biol. 2015;60:1346-55. https://doi.org/10.1016/j.archoralbio.2015.06.006, PMID:26126289

[36]Yoshimi H, Sasaguri K, Tamaki K, Sato S. Identification of the occurrence and pattern of masseter muscle activities during sleep using EMG and accelerometer systems. Head Face Med 2009;11:5;7.

[37] Bakke M. Bite force and occlusion. Semin Orthod. 2006;12:120-6. https:// doi.org/10.1053/j.sodo.2006.01.005

[38] Hasegawa $Y$, Lavigne $G$, Rompré $P$, Kato $T$, Urade $M$, Huynh N . Is there a first night effect on sleep bruxism? A sleep laboratory study. J Clin Sleep Med. 2013;09:1139-45. https://doi.org/10.5664/jcsm.3152, PMID:24235894

[39] Colonna A, Segù M, Lombardo L, Manfredini D. Frequency of sleep bruxism behaviors in healthy young adults over a four-night recording span in the home environment. Appl Sci (Basel). 2020;11:195. https://doi.org/10.3390/ app11010195

[40] Ruben JL, Roeters FJM, Truin GJ, Loomans BAC, Huysmans MCDNJM. Cup-shaped tooth wear defects: more than erosive challenges? Caries Res. 2019;53:467-74. https://doi.org/10.1159/000496983, PMID:30840963

[41] Tantbirojn D, Pintado MR, Versluis A, Dunn C, Delong R. Quantitative analysis of tooth surface loss associated with gastroesophageal reflux disease: a longitudinal clinical study. J Am Dent Assoc. 2012;143:278-85. https://doi. org/10.14219/jada.archive.2012.0153, PMID:22383209

[42] O'Toole S, Osnes C, Bartlett D, Keeling A. Investigation into the validity of WearCompare, a purpose-built software to quantify erosive tooth wear progression. Dent Mater. 2019;35:1408-14. https://doi.org/10.1016/j.dental.2019.07.023, PMID:31402133

[43] Etman MK, Woolford M, Dunne S. Quantitative measurement of tooth and ceramic wear: in vivo study. Int J Prosthodont. 2008;21:245-52. PMID:18548965

[44] Branco AC, Colaço R, Figueiredo-Pina CG, Serro AP. A State-of-the-Art Review on the Wear of the Occlusal Surfaces of Natural Teeth and Prosthetic Crowns. Materials (Basel). 2020;13:3525. https://doi.org/10.3390/ ma13163525, PMID:32785120

This is an open-access article distributed under the terms of Creative Commons Attribution-NonCommercial License 4.0 (CC BYNC 4.0), which allows users to distribute and copy the material in any format as long as credit is given to the Japan Prosthodontic Society. It should be noted however, that the material cannot be used for commercial purposes. 